\title{
The relaxation theory applied to optimal control problems of semilinear elliptic equations
}

\author{
Eduardo Casas \\ Dpto. de Matemática Aplicada y Ciencias de la Computación, E.T.S.I. \\ Industriales y de Telecomunicación, Universidad de Cantabria \\ Av. Los Castros s/n, 39071 Santander, Spain. Tel: 34-42-201427. \\ Fax: 34-42-201829. e-mail: casas@macc.unican.es
}

\begin{abstract}
The relaxation methods, such as described by Warga (1972), are applied to the study of state-constrained optimal control problems governed by semilinear elliptic equations. The main issue is to prove the convergence of the solutions of the discretized control problems to optimal controls of the relaxed continuous problem. In order to obtain this result we make a stability assumption of the optimal cost functional with respect to small perturbation of the feasible state set.
\end{abstract}

\section{Keywords}

Relaxed controls, state constraints, elliptic equations, finite element method

\section{INTRODUCTION}

In this paper, we are concerned with an optimal control problem of a semilinear elliptic equation, with control and state constraints. No convexity assumption is made on the feasible control set or the cost functional, therefore the existence of an optimal control can not be proved. However, after the discretization of the continuous problem (by using finite elements), we usually obtain a finite dimensional optimization problem having a solution. Then some questions arise: can we state any relation between these solutions and the continuous control problem?, do they converge to something in some topology when the dimension of the discretized problem is increased?

The reader is referred to Chryssoverghi (1985), (1986) and Chryssoverghi and Kokkinis (1994) for some related papers. We try to improve the existing theory by assuming less restrictive conditions on the problem (mainly on the state equation) and by including pointwise constraints in the control problem. On the other hand, in order to prove the convergence of the discretization of a state-constrained optimal control problem, it is usually required to enlarge the set of feasible states. Hereafter we will give a stability condition for the control problem in such a way that for the stable problems is not neces- 
sary to enlarge the set of feasible states to achieve the convergence of the discretizations. Moreover, we prove that almost all problems are stable; see Casas (1992).

\section{THE OPTIMAL CONTROL PROBLEM}

Let $\Omega$ be an open bounded subset of $\mathbb{R}^{n}, n=2$ or 3 , with a Lipschitz boundary. To simplify the presentation we will also assume that $\Omega$ is convex. However this assumption can be removed if we assume $\Gamma=\partial \Omega$ to be of class $C^{1,1}$, we will come back to this issue below. In $\Omega$ we consider the operator

$A y=-\sum_{i, j=1}^{n} \partial_{x_{j}}\left[a_{i j}(x) \partial_{x_{i}} y\right]$

where $a_{i j} \in C^{0,1}(\bar{\Omega}), 1 \leq i, j \leq n$ and satisfy the usual ellipticity assumption

$\exists \lambda>0$ such that $\sum_{i, j=1}^{n} a_{i j}(x) \xi_{i} \xi_{j} \geq \lambda\|\xi\|^{2} \quad \forall x \in \Omega$ and $\forall \xi \in \mathbb{R}^{n}$.

Let $\mathcal{K}$ be a compact subset of $\mathbb{R}^{m}, m \geq 1$, and let $f: \Omega \times(\mathbb{R} \times \mathcal{K}) \longrightarrow \mathbb{R}$ be a Carathèdory function, monotone non increasing with respect to the second variable and satisfying

$\left\{\begin{array}{l}\exists \psi_{0} \in L^{2}(\Omega) \text { such that }|f(x, 0, u)| \leq \psi_{0}(x) \quad \forall u \in \mathcal{K} \text { and a.e. } x \in \Omega, \\ \forall M>0 \exists \psi_{M} \in L^{2}(\Omega) \text { such that } \\ \left|f\left(x, y_{2}, u\right)-f\left(x, y_{1}, u\right)\right| \leq \psi_{M}(x)\left|y_{2}-y_{1}\right| \text { a.e. } x \in \Omega, \forall\left|y_{1}\right|,\left|y_{2}\right| \leq M, \forall u \in \mathcal{K} .\end{array}\right.$

Let us denote by $\mathcal{U}$ the set of measurable functions $u: \Omega \longrightarrow \mathcal{K}$. For each $u \in \mathcal{U}$ we consider the state equation

$\left\{\begin{array}{l}A y=f(x, y(x), u(x)) \text { in } \Omega, \\ y=0 \text { on } \Gamma .\end{array}\right.$

The next theorem claims the well posedness of the state equation.

Theorem 1 Under the previous assumptions (2) has a unique solution $y_{u}$ in $H_{0}^{1}(\Omega) \cap$ $H^{2}(\Omega)$. Moreover there exists a constant $C_{\mathcal{K}}>0$ such that

$\left\|y_{u}\right\|_{H^{2}(\Omega)} \leq C_{\mathcal{K}} \quad \forall u \in \mathcal{U}$

This theorem can be proved by classical arguments. First we can consider $f$ bounded and use Schauder's fixed point theorem to deduce the existence of a solution in $H^{1}(\Omega)$, 
the uniqueness being a consequence of the monotonicity of $f$ w.r.t. the second variable. If $f$ is not bounded, we can define

$$
f_{M}(x, y, u)=\left\{\begin{array}{cl}
+M & \text { if } y>+M \\
-M, & \text { if } y<-M \\
f(x, y, u) & \text { otherwise }
\end{array}\right.
$$

Thus we have a unique solution $y_{M}$ for every $M$. Then using again the monotonicity of $f$ and (1), we can obtain the boundedness $y_{M}$ in $\Omega$ independently of $M$ by Stampachia's method (1965). Thus $f_{M}\left(x, y_{M}(x), u(x)\right)=f\left(x, y_{M}(x), u(x)\right)$ for $M$ large enough, which implies that $y_{M}$ is a solution of $(2)$ in $H^{1}(\Omega) \cap L^{\infty}(\Omega)$, the uniqueness in this space is once more a consequence of the monotonicity of $f$ w.r.t. $y$. Finally, the $H^{2}(\Omega)$-regularity follows from the convexity of $\Omega$ and the Lipschitz regularity of the coefficients $a_{i j}$; see Grisvard (1985).

Remark 1 In the case of a nonconvex set $\Omega$, the previous theorem remains to be true under the $C^{1,1}$-regularity of $\Gamma$. Indeed the unique modification in the previous argumentation arises in the proof of the $H^{2}(\Omega)$-regularity of the solution. This regularity is still true when the convexity assumption of $\Omega$ is replaced by the $C^{1,1}$-regularity of $\Gamma$; see Grisvard (1985). It is known that the $C^{1}$-regularity of $\Gamma$ is not enough to assure the $H^{2}(\Omega)$-regularity of $y$; see Jerison and Kenig (1994).

Now we consider a Carathéodry function $L: \Omega \times(\mathbb{R} \times \mathcal{K}) \longrightarrow \mathbb{R}$ satisfying

$\forall M>0 \quad \exists \phi_{M} \in L^{1}(\Omega)$ such that $|L(x, y, u)| \leq \phi_{M}(x)$ a.e. $x \in \Omega, \forall|y| \leq M, \forall u \in \mathcal{K}$.(4)

Now we can state the optimal control problem as follows

$\left(\mathrm{P}_{\delta}\right)\left\{\begin{array}{l}\text { Minimize } J(u)=\int_{\Omega} L\left(x, y_{u}(x), u(x)\right) d x, \\ u \in \mathcal{U}, g\left(x, y_{u}(x)\right) \leq \delta \forall x \in \bar{\Omega},\end{array}\right.$

where $\delta \in \mathbb{R}$ and $g: \bar{\Omega} \times \mathbb{R} \longrightarrow \mathbb{R}$ is a continuous function.

We finish this section introducing the stability concept of $\left(\mathrm{P}_{\delta}\right)$ w.r.t. perturbations of the set of feasible states.

Definition 1 We will say that $\left(\mathrm{P}_{\delta}\right)$ is stable to the right if

$\lim _{\delta^{\prime} \backslash \delta} \inf \left(\mathrm{P}_{\delta^{\prime}}\right)=\inf \left(\mathrm{P}_{\delta}\right)$

Analogously, $\left(\mathrm{P}_{\delta}\right)$ is stable to the left if

$\lim _{\delta^{\prime} / \delta} \inf \left(\mathbf{P}_{\delta^{\prime}}\right)=\inf \left(\mathbf{P}_{\delta}\right)$

$\left(\mathrm{P}_{\delta}\right)$ is said stable if it is stable to the left and to the right simultaneously. 
The next theorem establishes how often this stability condition is satisfied.

Theorem 2 There exists $\delta_{0} \in \mathbb{R}$ such that $\left(\mathrm{P}_{\delta}\right)$ has no feasible control for $\delta<\delta_{0}$. For every $\delta>\delta_{0}$, except at most a countable number of them, problem $\left(\mathrm{P}_{\delta}\right)$ is stable.

Proof. From Theorem 1 we know the existence of a constant $M>0$ such that $\left|y_{u}(x)\right| \leq M$ for all $x \in \Omega$ and $u \in \mathcal{U}$. Let us set $\lambda_{M}$ and $\Lambda_{M}$ the minimum and maximum of $g$ over $\bar{\Omega} \times[-M,+M]$. Then it is obvious that $\left(\mathrm{P}_{\delta}\right)$ has no feasible control for $\delta<\lambda_{M}$, while every element of $\mathcal{U}$ is a feasible control for every $\delta \geq \Lambda_{M}$. Let us set $\delta_{0}=\inf \{\delta$ : $\left(\mathrm{P}_{\delta}\right)$ has at least one feasible control $\}$. Then $\lambda_{M} \leq \delta_{0} \leq \Lambda_{M}$.

Finally we prove that $\left(\mathrm{P}_{\delta}\right)$ is stable for almost all $\delta>\delta_{0}$. Let us consider the function $h:\left(\delta_{0},+\infty\right) \longrightarrow \mathbb{R}$ defined by $h(\delta)=\inf \left(\mathrm{P}_{\delta}\right)$. Then $h$ is a monotone non increasing function and then continuous at every point $\delta$, except at most a countable number of them. The theorem follows from the fact that the continuity of $h$ in $\delta$ is equivalent to the stability of $\left(\mathrm{P}_{\delta}\right)$.

\section{THE RELAXED CONTROL PROBLEM}

In this section we apply the relaxation theory to immerse the control set $\mathcal{U}$ in a bigger class of controls such that the new control problem has at least one solution. To do this we follow the approach described by Warga (1972). As usual $C(\mathcal{K})$ denotes the space of continuous functions endowed with the maximum norm and $M(\mathcal{K})=C(\mathcal{K})^{*}$ is the space of real regular Borel measures in $\mathcal{K}$. We also set $\mathcal{P}(\mathcal{K})$ equal to the subset of $M(\mathcal{K})$ formed by the probability measures in $\mathcal{K}$. With $\mathcal{R}$ we denote the subset of the Banach space $L^{\infty}(\Omega, M(\mathcal{K}))=L^{1}(\Omega, C(\mathcal{K}))^{*}$ formed by the functions $r$ such that $r(x) \in \mathcal{P}(\mathcal{K})$ for almost all $x \in \Omega$. Given a Carathéodory function $\phi: \Omega \times \mathcal{K} \longrightarrow \mathbb{R}$, we denote by $\phi_{R}: \Omega \times M(\mathcal{K}) \longrightarrow \mathbb{R}$ the function defined by

$\phi_{R}(x, \mu)=\int_{\mathcal{K}} \phi(x, k) d \mu(k)$

In some sense, $\phi_{R}$ can be considered as an extension of $\phi$, simply by taken $\phi(x, k)=$ $\dot{\phi}_{R}\left(x, \delta_{[k]}\right)$, where $\delta_{[k]}$ is the Dirac measure centered at the point $k$ of $\mathcal{K}$. Consequently, we can define the functions $f_{R}, L_{R}: \Omega \times \mathbb{R} \times M(\mathcal{K}) \longrightarrow \mathbb{R}$ by

$f_{R}(x, y, \mu)=\int_{\mathcal{K}} f(x, y, k) d \mu(k)$ and $L_{R}(x, y, \mu)=\int_{\mathcal{K}} L(x, y, k) d \mu(k)$

Now we define the relaxed control problem in the following way

$\left(\operatorname{RP}_{\delta}\right)\left\{\begin{array}{l}\text { Minimize } J_{R}(r)=\int_{\Omega} L_{R}\left(x, y_{r}(x), r(x)\right) d x \\ r \in \mathcal{R}, g\left(x, y_{r}(x)\right) \leq \delta \forall x \in \bar{\Omega},\end{array}\right.$ 
with $y_{r} \in H_{0}^{1}(\Omega) \cap H^{2}(\Omega)$ being the solution of the problem

$\left\{\begin{array}{l}A y=f_{R}(x, y(x), r(x)) \text { in } \Omega, \\ y=0 \text { on } \Gamma .\end{array}\right.$

Let us remark that $\mathcal{U}$ can be considered as a subset of $\mathcal{R}$ by identifying $u(x)$ and $r(x)=\delta_{[u(x)]}$. Moreover, with this identification we have $J_{R}(r)=J(u), y_{r}=y_{u}$ and $f_{R}\left(x, y_{r}(x), r(x)\right)=f\left(x, y_{u}(x), u(x)\right)$. On the other hand $\mathcal{U}$ is dense in $\mathcal{R}$; see Warga (1972). Therefore $\left(\mathrm{RP}_{\delta}\right)$ can be considered as an extension of $\left(\mathrm{P}_{\delta}\right)$. Furthermore $\left(\mathrm{RP}_{\delta}\right)$ has at least one solution as we will prove below. Then a natural question is whether $\inf \left(\mathrm{RP}_{\delta}\right)=\inf \left(\mathrm{P}_{\delta}\right)$. Here we have the answer

Theorem 3 Let $\delta_{0}$ be as in Theorem 2. $\left(\mathrm{RP}_{\delta}\right)$ has at least one solution for every $\delta>\delta_{0}$. Moreover $\inf \left(\mathrm{RP}_{\delta}\right)=\inf \left(\mathrm{P}_{\delta}\right)$ if and only if $\left(\mathrm{P}_{\delta}\right)$ is stable to the right.

Proof. It is well known that $\mathcal{R}$ is convex, metrizable (with coinciding metric and weak ${ }^{\star}$ topologies) and compact. Then the set of feasible relaxed controls is compact too. Moreover this set is not empty due to the fact that $\delta>\delta_{0}$. Finally, the continuity of $J_{R}$ on $\mathcal{R}$ allows to conclude the existence of an optimal relaxed control.

To prove the second part of the theorem we first state the following inequalities

$\inf \left(\mathrm{RP}_{\delta^{\prime}}\right) \leq \inf \left(\mathrm{P}_{\delta^{\prime}}\right) \leq \inf \left(\mathrm{RP}_{\delta}\right) \leq \inf \left(\mathrm{P}_{\delta}\right)$ for every $\delta^{\prime}>\delta$.

The first and the last inequalities are a consequence of the identification of every feasible control for $\left(\mathrm{P}_{\delta}\right)$ (resp. $\left(\mathrm{P}_{\delta}^{\prime}\right)$ ) with a feasible control for $\left(\mathrm{RP}_{\delta}\right)\left(\right.$ resp. $\left.\left(\mathrm{RP}_{\delta}^{\prime}\right)\right)$. Let us prove the second inequality. If $r$ is a feasible control for $\left(\mathrm{RP}_{\delta}\right)$, then we can take a sequence of controls $\left\{u_{k}\right\}_{k=1}^{\infty}$ such that $r_{k}(x)=\delta_{\left[u_{k}(x)\right]} \rightarrow r(x)$ weakly ${ }^{\star}$ in $L^{\infty}(\Omega, M(\mathcal{K}))$. Since $y_{u_{k}} \rightarrow y_{r}$ uniformly in $\bar{\Omega}$, then $\delta \geq g\left(x, y_{r}(x)\right)=\lim _{k \rightarrow \infty} g\left(x, y_{u_{k}}(x)\right)$, therefore $g\left(x, y_{u_{k}}(x)\right) \leq \delta^{\prime}$ for every $x \in \bar{\Omega}$ and $k$ bigger than a certain $k_{0}$, only depending on $\delta^{\prime}$. Thus the controls $\left\{u_{k}\right\}_{k \geq k_{0}}$ are feasible for problem $\left(\mathbf{P}_{\delta^{\prime}}\right)$ and

$J_{R}(r)=\lim _{k \rightarrow \infty} J\left(u_{k}\right) \geq \inf \left(\mathrm{P}_{\delta^{\prime}}\right)$,

which leads to the desired inequality.

Finally, the proof will be concluded by proving that $\lim _{\delta^{\prime} \searrow_{\delta} \delta} \inf \left(\mathrm{RP}_{\delta^{\prime}}\right)=\inf \left(\mathrm{RP}_{\delta}\right)$. Let $r_{\delta^{\prime}}$ be a solution of $\left(\mathrm{RP}_{\delta^{\prime}}\right)$ for every $\delta^{\prime}>\delta$. Using the compactness of $\mathcal{R}$, we can take a sequence $\left\{r_{\delta_{j}}\right\}_{j=1}^{\infty}$, with $\delta_{j} \searrow \delta$, such that $r_{\delta_{j}} \rightarrow r$ weakly ${ }^{\star}$ for some $r \in \mathcal{R}$. Using the uniform convergence $y_{r_{\delta_{j}}} \rightarrow y_{r}$ in $\bar{\Omega}$, we obtain

$g\left(x, y_{r}(x)\right)=\lim _{j \rightarrow \infty} g\left(x, y_{r_{\delta_{j}}}(x)\right) \leq \lim _{j \rightarrow \infty} \delta_{j}=\delta$,

for every $x \in \bar{\Omega}$, Therefore $r$ is a feasible control for $\left(\mathrm{RP}_{\delta}\right)$. Thus we have

$\inf \left(\mathrm{RP}_{\delta}\right) \leq J_{R}(r)=\lim _{j \rightarrow \infty} J_{R}\left(r_{\delta_{j}}\right)=\lim _{\left.\delta^{\prime}\right\rangle_{\searrow}} \inf \left(\mathrm{RP}_{\delta^{\prime}}\right) \leq \inf \left(\mathrm{RP}_{\delta}\right)$ 
Corollary 1 If problem $\left(\mathrm{P}_{\delta}\right)$ is stable to the right and it has a solution $\bar{u}$, then $\vec{r}(x)=$ $\delta_{[\bar{u}(x)]}$ is also a solution of $\left(\mathrm{RP}_{\delta}\right)$.

\section{NUMERICAL APPROXIMATION OF THE CONTROL PROBLEM}

In this section we consider the numerical discretization of problem $\left(\mathrm{P}_{\delta}\right)$. As we will see below, the discrete problems have at least one solution, the natural question is to relate these optimal discrete controls with the continuous problem. Since the existence of a solution of $\left(\mathrm{P}_{\delta}\right)$ can not be stated under our assumptions, we look at the relaxed control problem, which has a solution. We will prove that the optimal discrete controls converge to optimal relaxed controls in some topology.

Let $\left\{\mathcal{T}_{h}\right\}_{h>0}$ be a regular family of triangulations in $\bar{\Omega}$ satisfying the inverse assumption; see Ciarlet (1978). Let us take $\bar{\Omega}_{h}=\cup_{T \in T_{h}} T, \Omega_{h}$ its interior and $\Gamma_{h}$ its boundary. Then we assume that $\Omega_{h}$ is convex and the vertices of $\mathcal{T}_{h}$ placed on the boundary $\Gamma_{h}$ are points of $\Gamma$. To every boundary triangle $T$ of $\mathcal{T}_{h}$ we associate another triangle $\tilde{T} \subset \bar{\Omega}$ with two interior sides to $\Omega$ coincident with two sides of $T$ and the third side is the curvilinear arc of $\Gamma$ limited by the other two sides. We denote by $\tilde{\mathcal{T}}_{h}$ the family formed by these boundary triangles with a curvilinear side and the interior triangles to $\Omega$ of $\mathcal{T}_{h}$, so $\bar{\Omega}=U_{T \in \bar{T}_{h}} T$. Now let us consider the spaces

$\mathcal{U}_{h}=\left\{u_{h} \in \mathcal{U}:\left.u_{h}\right|_{T}\right.$ is constant $\left.\forall T \in \tilde{\mathcal{T}}_{h}\right\}$

$V_{h}=\left\{y_{h} \in C(\bar{\Omega}):\left.y_{h}\right|_{T} \in \mathcal{P}_{1} \forall T \in \mathcal{T}_{h}\right.$ and $\left.y_{h}(x)=0 \quad \forall x \in \bar{\Omega} \backslash \Omega_{h}\right\}$

where $\mathcal{P}_{1}$ is the space of the polynomials of degree less than or equal to 1 . It is obvious that $V_{h} \subset H_{0}^{1}(\Omega)$. For each $u_{h} \in \mathcal{U}_{h}$ we denote by $y_{h}\left(u_{h}\right)$ the unique element of $V_{h}$ that satisfies:

$\int_{\Omega_{h}} \sum_{i, j=1}^{n} a_{i j}(x) \partial_{x_{i}} y_{h}\left(u_{h}\right)(x) \partial_{x_{j}} z_{h}(x) d x=\int_{\Omega_{h}} f\left(x, y_{h}\left(u_{h}\right)(x), u_{h}(x)\right) z_{h}(x) d x \quad \forall z_{h} \in V_{h}$.

Now we formulate the finite dimensional optimal control problem:

$\left(\mathrm{P}_{\delta h}\right)\left\{\begin{array}{l}\text { minimize } J_{h}\left(u_{h}\right)=\int_{\Omega_{h}} L\left(x, y_{h}\left(u_{h}\right)(x), u_{h}(x)\right) d x \\ \text { subject to } u_{h} \in \mathcal{U}_{h} \text { and } g\left(x_{j}, y_{h}\left(u_{h}\right)\left(x_{j}\right)\right) \leq \delta \quad 1 \leq j \leq n(h),\end{array}\right.$

where $\left\{x_{j}\right\}_{j=1}^{n(h)}$ is the set of vertices of $\mathcal{T}_{h}$. The following theorem state the existence of a solution for $\left(\mathrm{P}_{\delta h}\right)$.

Theorem 4 For every $\delta>\delta_{0}$ there exists $h_{\delta}>0$ such that $\left(\mathrm{P}_{\delta h}\right)$ has at least one solution $\bar{u}_{h}$ for all $h \leq h_{\delta}$. 
Proof. Since $\mathcal{U}_{h}$ is a compact set and $J_{h}$ is continuous, the existence of a solution of $\left(\mathrm{P}_{\delta h}\right)$ will be assured if we prove that the set of feasible controls is nonempty. Let $u_{0} \in \mathcal{U}$ be a feasible control for $\left(\mathrm{P}_{\delta_{0}}\right)$ and let us take $u_{0 h} \in \mathcal{U}_{h}$ such that $u_{0 h}(x) \rightarrow u_{0}(x)$ for almost every point $x \in \Omega$ as $h \rightarrow 0$. Then $y_{h}\left(u_{0 h}\right) \rightarrow y_{u_{0}}$ uniformly in $\bar{\Omega}$. Since $g\left(x, y_{u_{0}}(x)\right) \leq \delta_{0}$ for every $x \in \bar{\Omega}$, from the uniform convergence and the inequality $\delta>\delta_{0}$ we deduce the existence of $h_{\delta}>0$ such that the inequality $g\left(x, y_{h}\left(u_{0 h}\right)(x)\right) \leq \delta$ holds for all $x \in \bar{\Omega}$ and each $h \leq h_{\delta}$. Therefore $u_{0 h}$ is a feasible control for $\left(\mathrm{P}_{\delta h}\right)$, which completes the proof.

Finally we prove the convergence result of the numerical approximation.

Theorem 5 Let us assume that $\left(\mathbf{P}_{\delta}\right)$ is stable and let $h_{\delta}>0$ be as in Theorem 4. Given a family of controls $\left\{\bar{u}_{h}\right\}_{h<h_{\delta}}, \bar{u}_{h}$ being a solution of $\left(\mathbf{P}_{\delta h}\right)$, there exist subsequences $\left\{\bar{u}_{h_{k}}\right\}_{k \in N}$, with $h_{k} \rightarrow 0$ as $k \rightarrow \infty$, and elements $\bar{r} \in \mathcal{R}$ such that $\bar{r}_{h_{k}}(x)=\delta_{\left[\bar{u}_{h_{k}}(x)\right]} \rightarrow \bar{r}$ in the weak topology of $L^{\infty}(\Omega, M(\mathcal{K}))$. Each one of these limit points is a solution of $\left(\mathrm{RP}_{\delta}\right)$. Moreover we have

$\lim _{h \rightarrow 0} J_{h}\left(\bar{u}_{h}\right)=\inf \left(\mathrm{RP}_{\delta}\right)=\inf \left(\mathbf{P}_{\delta}\right)$

Proof. Let $\bar{y}_{h}$ be the state associated to $\bar{u}_{h}$ and let us write $\bar{r}_{h}(x)=\delta_{\left[\bar{u}_{h_{k}}(x)\right]}$. Since $\left\{\bar{r}_{h}\right\}_{h \leq h_{\delta}} \subset \mathcal{R}$ and $\mathcal{R}$ is a weak ${ }^{\star}$ compact metrizable subset of the space $L^{\infty}(\Omega, M(\mathcal{K}))$, we can extract a subsequence $\left\{\bar{r}_{h_{k}}\right\}$ such that $h_{k} \rightarrow 0$ and $\bar{r}_{h_{k}}(x)=\delta_{\left[\bar{u}_{h_{k}}(x)\right]} \rightarrow \bar{r}$ weakly* in $L^{\infty}(\Omega, M(\mathcal{K}))$ for some element $\bar{r} \in \mathcal{R}$. Now we prove that $\bar{r}$ is a solution of $\left(\mathrm{RP}_{\delta}\right)$. Let $\dot{\bar{y}}$ be the state associated to $\bar{r}$. Since $\bar{y}_{h_{k}} \rightarrow \bar{y}$ uniformly in $\bar{\Omega}$ and $g\left(x_{j}, \bar{y}_{h_{k}}\left(x_{j}\right)\right) \leq \delta$ for every $1 \leq j \leq n(h)$, it follows that $g(x, \bar{y}(x)) \leq \delta$ and therefore $\bar{r}$ is a feasible control for the problem $\left(\mathrm{RP}_{\delta}\right)$.

Let $\delta^{\prime} \in\left[\delta_{0}+\epsilon, \delta\right)$, with $0<\epsilon<\delta-\delta_{0}$ fixed, and let $r_{\delta^{\prime}}$ be a solution of $\left(\mathrm{RP}_{\delta^{\prime}}\right)$. Since $\mathcal{U}$ is dense in $\mathcal{R}$, we can take sequence $\left\{u^{j}\right\}_{j=1}^{\infty} \subset \mathcal{U}$ such that $u^{j} \rightarrow r_{\delta^{\prime}}$ weakly ${ }^{\star}$ in $L^{\infty}(\Omega, M(\mathcal{K}))$. Taking into account the uniform convergence $y_{u^{j}} \rightarrow y_{r_{\sigma^{\prime}}}$, we deduce the existence of $j_{\delta^{\prime}} \in N$ such that $g\left(x, y_{u^{j}}(x)\right) \leq \delta^{\prime}+\epsilon / 2$ for every $x \in \bar{\Omega}$ and $j \geq j_{\delta^{\prime}}$. For each $j$ fixed we take a sequence $\left\{u_{h}\right\}_{h>0}$, with $u_{h} \in \mathcal{U}_{h}$ and such that $u_{h}(x) \rightarrow$ $u^{j}(x)$ for almost all point $x \in \Omega$. From the uniform convergence $y_{h}\left(u_{h}\right) \rightarrow y_{u}$ and the inequality $g\left(x, y_{u^{j}}(x)\right) \leq \delta^{\prime}+\epsilon / 2<\delta$ for all $x \in \bar{\Omega}$ we deduce the existence of $h^{j}$ such that $g\left(x, y_{h}\left(u_{h}\right)(x)\right) \leq \delta \forall x \in \bar{\Omega}$ and $\forall h \leq h^{j}$. Hence $u_{h}$ is a feasible control for $\left(\mathrm{P}_{\delta h}\right)$ always that $h \leq h^{j}$. Then, we get $J_{h_{k} R}\left(\bar{r}_{h_{k}}\right)=J_{h_{k}}\left(\bar{u}_{h_{k}}\right) \leq J_{h_{k}}\left(u_{h_{k}}\right)$ whenever $h_{k} \leq h^{j}$. Thus we have

$J_{R}(\bar{r})=\lim _{k \rightarrow \infty} J_{h_{k} R}\left(\bar{r}_{h_{k}}\right) \leq \lim _{k \rightarrow \infty} J_{h_{k}}\left(u_{h_{k}}\right)=J\left(u^{j}\right)$

Now taking the limit when $j \rightarrow \infty$, we obtain that $J_{R}(\bar{r}) \leq J_{R}\left(r_{\delta^{\prime}}\right)$. Finally, the feasibility of $\bar{r}$ for $\left(\mathrm{RP}_{\delta}\right)$ and the stability condition (Definition 1) enables us to conclude that

$\inf \left(\mathrm{RP}_{\delta}\right) \leq J_{R}(\bar{r}) \leq \lim _{\delta^{\prime} / \delta} J_{R}\left(r_{\delta^{\prime}}\right)=\lim _{\delta^{\prime} / \delta} \inf \left(\mathrm{RP}_{\delta^{\prime}}\right) \leq \lim _{\delta^{\prime} / \delta} \inf \left(\mathrm{P}_{\delta^{\prime}}\right)=\inf \left(\mathrm{P}_{\delta}\right)=\inf \left(\mathrm{RP}_{\delta}\right)$,

which proves that $\bar{r}$ is a solution of $\left(\mathrm{RP}_{\delta}\right)$. The rest of theorem is immediate. 


\section{ACKNOWLEDGEMENT}

The author would like to thank Dirección General de Investigación Cientifica y Técnica (Spain) for its support to this research.

\section{REFERENCES}

Casas, E. (1992) Finite element approximations for some state-constrained optimal control problems, in Mathematics of the Analysis and Design in Process Control (eds. P. Borne, S. Tzafestas, and N. Radhy), Amsterdam, North Holland.

Chryssoverghi, I. (1985) Numerical approximation of nonconvex optimal control problems defined by parabolic equations. J. Optim. Theory Appl., 45, 73-88.

Chryssoverghi, I. (1986) Nonconvex optimal control of nonlinear monotone parabolic systems. Systems Control Lett., 8, 55-62.

Chryssoverghi, I. and Kokkinis, B. (1994) Discretization of nonlinear elliptic optimal control problems. Systems Control Lett., 22, 227-34.

Ciarlet, P.G. (1978) The Finite Element Method for Elliptic Problems. North-Holland, Amsterdam.

Grisvard, P. (1985) Elliptic Problems in Nonsmooth Domains. Pitman, Boston-LondonMelbourne.

Jerison, D. and Kenig, C. (1995) The inhomogeneous Dirichlet problem in Lipschitz domains. To appear.

Stampacchia, G. (1965) Le problème de Dirichlet pour les équations elliptiques du second ordre à coefficients discontinus. Ann. Inst. Fourier (Grenoble), 15, 198-258.

Warga, J. (1972) Optimal control of differential and functional equations. Academic Press, New York. 